\title{
Anestesia de um Graxaim-do-campo (Pseudalopex gymnocercus) com propofol por infusão contínua
}

\author{
Pampas fox (Pseudalopex gymnocercus) anesthesia with propofol by continuous infusion \\ Giordano Cabral Gianotti ${ }^{1}$, Wanessa Krüger Beheregaray², Fernanda Silveira Nóbrega², Márcio Poletto \\ Ferreira ${ }^{3}$, J osé Pedro Abati Viana Rocha ${ }^{4}$, Ingrid Vera Stein ${ }^{4}$, Emerson Antonio Contesini ${ }^{5}$, Marcelo \\ Meller Alievi ${ }^{5} \&$ Marcelo de Souza Muccillo $^{6}$
}

\begin{abstract}
RESUMO
Graxaim-do-campo (Pseudalopex gymnocercus) é um canídeo silvestre que habita os campos da região Sul do Brasil. Sabe-se muito pouco sobre anestesia nesta espécie, mas acredita-se ser semelhante à usada em cães domésticos. $\mathrm{O}$ propofol, amplamente usado em cães, é um anestésico injetável distinto das outras drogas anestésicas, que proporciona rápida indução, ótima recuperação, com mínimo efeito residual. Nesse estudo, foi usado propofol em infusão contínua para remoção de implante ósseo em um graxaim de 4,5kg. Antes de iniciar a anestesia geral, utilizou-se como pré-medicação tiletamina- zolazepam, cloridrato de petidina e citrato de fentanila, todos pela via intramuscular. Com a tranqüilização e contenção, realizou-se a avaliação pré-anestésica do animal. O paciente foi induzido com propofol a uma dose de $4 \mathrm{mg} / \mathrm{kg}$, IV, até se obter o efeito anestésico desejado. Imediatamente após a indução, iniciou-se infusão contínua de propofol a uma dose de $0,35 \mathrm{mg} / \mathrm{kg} / \mathrm{h}$ para a manutenção. O procedimento anestésico durou uma hora e vinte minutos, tendo parâmetros, como reflexo palpebral, frequiência cardíaca, freqüência respiratória, pressão arterial média e oximetria, avaliados de dez em dez minutos. O objetivo deste relato constistiu em avaliar alterações cardiovasculares e respiratórias do protocolo utilizado, bem como a funcionalidade da técnica. A diminuição da pressão arterial média e depressão respiratória foram marcantes no procedimento, mas a técnica mostrou-se viável e as doses do fármaco foram efetivas e adequadas, apesar de necessitar de estrutura e aparelhagem específicas.
\end{abstract}

Descritores: Graxaim-do-Campo, Propofol, Infusão Contínua, Anestesia Venosa Total

\section{ABSTRACT}

Pampas fox (Pseudalopex gymnocercus) is a Wild-life canid that lives in the South Region of Brazil. It is known very little about this kind of species anesthesia, but it is believed to be similar to that used in domestic dogs. The propofol, widely used in dogs, is an injectable anesthetic distinct from other anesthetic drugs, which provides rapid induction, good recovery with minimum residual effect. In this study, propofol was used by continuous infusion for bone implant removal in a $4.5 \mathrm{~kg}$ graxaim. Before starting the general anesthesia it was used as pre-medication tiletamine-zolazepam, pethidine hydrochloride and fentanil citrate, all by intramuscular. After tranquilization and containment, there was made a pre-anesthetic evaluation of the animal. The patient was induced with a dose of $4 \mathrm{mg} / \mathrm{kg}$, IV propofol to obtain the desired anesthetic effect. Immediately after the induction, began continuous infusion of propofol at a $0.35 \mathrm{mg} / \mathrm{kg} / \mathrm{h}$ dose for maintenance. The anesthetic procedure lasted one hour and twenty minutes, with parameters, ocular reflex, heart rate, respiratory frequency, mean arterial pressure and oximetry, was evaluated every ten minutes. The objective of this report was to assess changes in cardiovascular and respiratory in the protocol used, as well as the functionality of the technique. The decrease in the mean arterial pressure and respiratory depression were striking in the procedure, but the technique shown to be viable and the doses of the drug were effective and adequate, although they require a specific structure and equipment.

Keywords: Pampas Fox, Propofol, Continuous Infusion, Total Intravenous Anaesthesia 
INTRODUÇÃO

O graxaim-do-campo (P. gymnocercus), também conhecido como cachorro-do-mato, é um canídeo silvestre que habita os campos do sul do Brasil, Argentina, Uruguai e Paraguai. Com até $1,0 \mathrm{~m}$ de comprimento, peso entre 4,2 e $5.9 \mathrm{~kg}$, é acostumado à presença humana $[10,14]$.

Técnicas anestésicas em canídeos silvestres são semelhantes às usadas em cães domésticos, em que a anestesia com propofol, bem como as medicações pré-anestésicas, já são bastante difundidas e pesquisadas, assim como em outras espécies $[11,18]$. O propofol é um anestésico injetável - distinto das outras drogas anestésicas - que proporciona rápida indução, ótima recuperação com mínimo efeito residual, portanto, ideal em infusão contínua na anestesia venosa total (AVT). Possui efeitos adversos, como depressão respiratória e hipotensão [5,7,13,16,17]. A sua dose de infusão varia conforme o sinergismo com outras drogas na anestesia e/ou o estímulo cirúrgico [7].

A AVT é uma alternativa à anestesia inalatória, com o surgimento de anestésicos de rápido metabolismo, efeitos mais específicos e menor hepatoxidade, além de não poluir o ambiente das salas cirúrgicas. Existe, ainda, uma demanda por protocolos seguros para serem utilizados nas cirurgias de curta ou média duração $[12,18]$. A infusão contínua permite um controle melhor dos efeitos da droga, podendo-se manter a sua administração com maior grau de precisão, segurança e previsibilidade [4].

Nesse estudo, é relatado o uso do propofol em infusão contínua para cirurgia ortopédica no fêmur de um graxaim-do-campo, avaliando alterações cardiovasculares e respiratórias do protocolo, bem como a funcionalidade da técnica.

\section{RELATO DE CASO}

Um graxaim-do-campo, fêmea, pesando 4,5 $\mathrm{kg}$, foi anestesiado no Hospital de Clínicas Veterinárias da Universidade Federal do Rio Grande do Sul para remoção de uma placa de compressão dinâmica empregada na redução de uma fratura de fêmur. Como medicação pré-anestésica (MPA) optou-se pela administração de $3 \mathrm{mg} / \mathrm{kg}$ de tiletamina- zolazepam ${ }^{1}$, $2 \mathrm{mg} / \mathrm{kg}$ de cloridrato de petidina ${ }^{2}$ e $2 \mathrm{mcg} / \mathrm{kg}$ de citrato de fentanila ${ }^{3}$, todos pela via intramuscular.
Com o animal tranqüilo e contido, realizou-se a avaliação pré-anestésica, quando se observou freqüência cardíaca (FC) de 198 bpm, taquipnéia, mucosas normocoradas, tempo de preenchimento capilar (TPC) de 2s, boa hidratação, sem alteração nos linfonodos. Então, foi realizado o acesso venoso, mantendo-se com solução de ringer com lactato ${ }^{4}$, sendo que a velocidade de infusão foi variável no decorrer do procedimento. Também foi realizado o acesso arterial na artéria metatarsiana com cateter 22 para mensuração, através do manômetro, da pressão arterial média (PAM) que, no momento do acesso, apresentava-se em 90mmHg (Figura 1).

Após o preparo, o paciente foi induzido com propofol $^{5}$ a uma dose de $4 \mathrm{mg} / \mathrm{kg}$, IV, até se obter o efeito anestésico desejado, como a redução da atividade reflexógena e o relaxamento da musculatura mandibular, para a entubação orotraqueal, sendo utilizado o tubo endotraqueal tipo murphy, descartável, $\mathrm{n}^{\mathrm{o}} 4.5$, com balonete. Durante o procedimento, forneceu-se oxigênio a $200 \mathrm{ml} / \mathrm{kg} / \mathrm{min}$ em respiração espontânea. Imediatamente após a indução anestésica, iniciou-se infusão contínua de propofol a uma dose de $0,35 \mathrm{mg} / \mathrm{kg} / \mathrm{min}$ para a manutenção anestésica (Figura 2). A anestesia geral durou uma hora e vinte minutos, tendo os seguintes parâmetros avaliados e tomados como referência de dez em dez minutos: reflexo palpebral medial, posição do globo ocular, TPC, relaxamento muscular, FC, freqüência respiratória (FR), PAM e oximetria $\left(\mathrm{Sp} . \mathrm{O}_{2}\right)$. No decorrer da anestesia, o paciente foi mantido aquecido com colchão térmico. A tabela 1 demonstra os principais parâmetros avaliados e os fatos relevantes constatados durante o procedimento anestésico. A avaliação do plano anestésico foi procedida através da verificação da presença ou não do tônus dos músculos da mandíbula e pela averiguação da intensidade dos reflexos palpebral e corneano.

Vinte minutos após o término do procedimento anestésico, o paciente apresentou recuperação do reflexo de deglutição e, então, foi realizada a extubação, com o paciente consciente e deambulando uma hora após, completamente recuperado da anestesia. Para o pós-operatório imediato, o paciente recebeu cloridrato de petidina na dose de $2 \mathrm{mg} / \mathrm{kg}$ e cetoprofeno $^{6}$ na dose de $1 \mathrm{mg} / \mathrm{kg}$. 


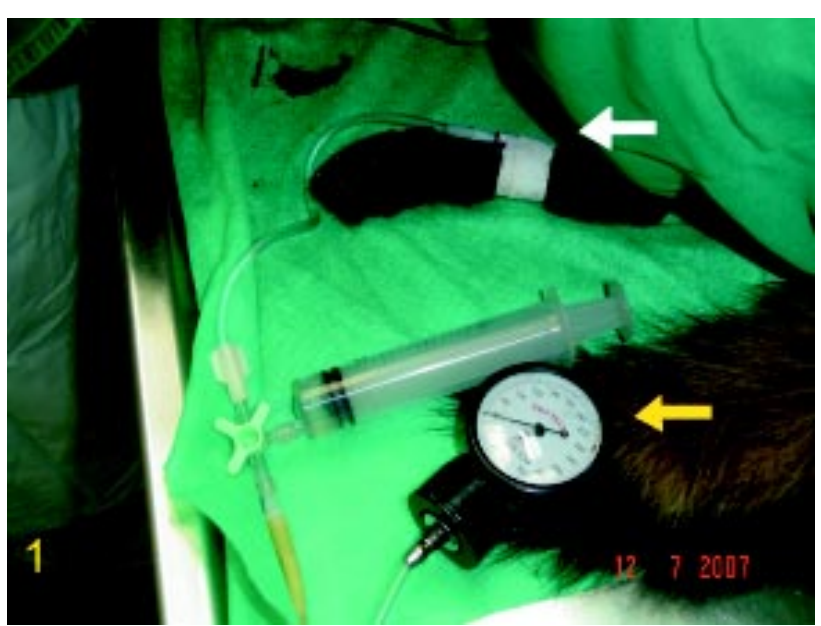

Figura 1. Anestesia graxaim-do-campo. Mensuração da PAM na artéria metatarsiana (Seta branca) através de manômetro (Seta amarela).

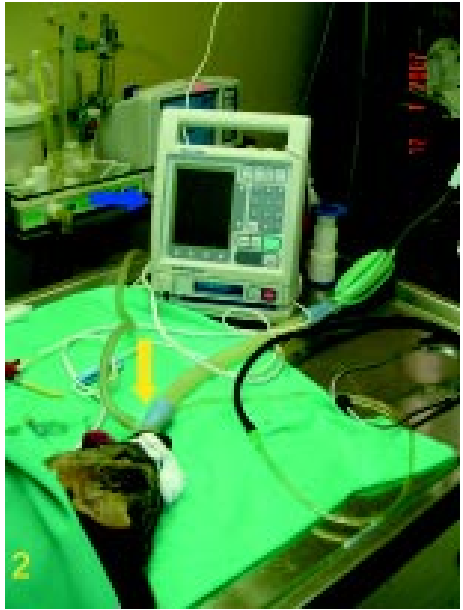

Figura 2. Anestesia graxaim-do-campo. Bomba de infusão (Seta azul) para manutenção da AVT e fornecimento de oxigênio a $100 \%$ através de sistema aberto (Seta amarela).

Tabela 1. Variação dos valores dos parâmetros pressão arterial média (PAM), freqüência cardíaca (FC), freqüência respiratória (FR), oximetria $\left(\mathrm{Sp} . \mathrm{O}_{2}\right.$ ) e velocidade do volume de reposição de fluidos (Fluído) durante o decorrer do procedimento anestésico.

\begin{tabular}{|c|c|c|c|c|c|c|c|c|c|}
\hline \multirow[b]{2}{*}{ Parâmetros } & \multicolumn{9}{|c|}{ Tempos } \\
\hline & $T 0^{*}$ & $T 1^{*}$ & $T 2^{*}$ & $T 3^{*}$ & $T 4^{*}$ & $T 5^{*}$ & $T 6^{*}$ & $T 7^{*}$ & $T 8^{*}$ \\
\hline$P A M(m m H g)$ & 80 & 60 & 50 & 60 & 60 & 90 & 90 & 80 & 70 \\
\hline$F C(b p m)$ & 100 & 108 & 113 & 108 & 112 & 121 & 126 & 130 & 110 \\
\hline$F R$ (MPM) & 12 & 12 & 14 & 12 & 10 & 10 & 8 & 8 & 8 \\
\hline $\mathrm{Sp.O}_{2}(\%)$ & 97 & 96 & 97 & 95 & 93 & 92 & 95 & 93 & 96 \\
\hline Fluido $\mathrm{ml} / \mathrm{kg} / \mathrm{h}$ & 10 & 10 & 50 & 50 & 40 & 10 & 10 & 10 & 10 \\
\hline
\end{tabular}

*Em todos os momentos o paciente permanceu com relaxamento da musculatura da mandíbula e leve reflexo palpebral medial e globo ocular rotado.

TO - indução, intubação e início da infusão, T1 - 10 minutos após indução, T2 - 20 minutos após indução e início do procedimento, T3 - 30 minutos após indução, T4 - 40 minutos após a indução, T5 - 50 minutos após a indução, T6 - 60 minutos após a indução, T7 - 70 minutos após a indução, T8 - 80 minutos após a indução, término do procedimento e fim da infusão de propofol.

\section{DISCUSSÃO}

A escolha de um protocolo para anestesia no graxaim-do-campo foi baseada na disponibilidade de fármacos e equipamentos, bem como no domínio das técnicas de sua utilização, com base em extensa revisão bibliográfica. A medicação pré-anes-tésica deve fornecer uma sedação adequada para um primeiro contato com o paciente, assim como uma analgesia apropriada durante todo o procedimento cirúrgico [11]. A avaliação dos parâmetros basais do paciente só foi possível, então, após a tranquilização, por se tratar de um animal não domesticado. Tanto a tiletamina-zolazepan, cloridrato de petidina e citrato de fentanila são bastante difundidos e estudados, principalmente em cães, mostrando-se apropriadas para o uso na medicação pré-anestésica, fornecendo boa sedação e analgesia no transoperatório [5,11,13,17].

Após a indução para a entubação e a manutenção anestésica, foram avaliados parâmetros como relaxamento da musculatura da mandíbula e redução da intensidade dos reflexos palpebrais, que são considerados como referência à avaliação do plano anestésico [2,12]. O uso da técnica foi satisfatória durante todo o procedimento, pela não necessidade de suplementação de propofol, bem como pelo fato da dose de manutenção anestésica permanecer em acordo com o relatado na bibliografia pesquisada $[2,12,13]$.

O fármaco pode causar depressão respiratória e isso ficou evidente com a queda dos movimentos 
respiratórios e diminuição da $\mathrm{Sp} . \mathrm{O}_{2}$ no procedimento anestésico relatado. Mesmo assim, existe ainda correlação da depressão respiratória com a associação farmacológica de opióides, o que também pode ter colaborado, nesse caso, para tal fato. Recomendase, então, o suporte ventilatório aos pacientes, realizado com oxigênio $100 \%$ e comprovando-se sua eficácia através da oximetria $[1,2,7,13,15,16,17,18]$. A oximetria é considerada um ótimo método para informar como está ocorrendo a oxigenação sangüínea $[12,6]$.

Dentre as características do propofol são incluídas efeito inotrópico negativo, menor resistência vascular sistêmica, diminuição da atividade simpática, causando bradicardia [17]. O fármaco promove significativo grau de hipotensão e esse evento pode estar relacionado a diferentes doses de infusão, variações nas medicações pré-anestésicas e estímulos cirúrgicos $[7,13,16,17]$. Em contra partida, alguns autores perceberam em seus trabalhos que o grau de variação da PAM do anestésico não foi tão significativo $[11,14$, 15,12]. Não havendo outras justificativas prováveis, a hipotensão foi evidente e correlacionada ao uso do fármaco, sendo a queda da pressão arterial alterada conforme a dose de infusão do anestésico, o baixo estímulo cirúrgico, bem como variações na MPA. Citase, ainda, uma taquicardia compensatória à diminuição da pressão arterial, como se pôde comprovar no caso estudado $[1,16]$.

A fluidoterapia foi instalada com objetivo de reposição volêmica e correção da PAM em caso de hipotensão, uma das complicações mais comuns no transoperatório. Para correção da diminuição da pressão, utiliza-se uma taxa de fluído que pode chegar à $90 \mathrm{ml} / \mathrm{kg} / \mathrm{h}$. No caso de insucesso, opta-se, então, por agentes vasoativos e inotrópicos. No caso relatado, a correção da pressão arterial, através da reposição com solução de ringer com lactato, ocorreu com sucesso [5].

O propofol mostrou-se um fármaco adequado para esta técnica anestésica e também para esse caso, pois produziu uma ótima indução e recuperação tranquiila do paciente, bem como excelente relaxamento muscular durante todo procedimento cirúrgico $[2,5,7,9,13,15,16,17]$. As doses recomendadas para infusão contínua são as mais variadas e, geralmente, oscilam entre 0,15 e $0,4 \mathrm{mg} / \mathrm{kg} / \mathrm{min} \quad[1,2,7$, $9,12,13,18]$. Na dose utilizada, $0,35 \mathrm{mg} / \mathrm{kg} / \mathrm{min}$, para manutenção anestésica no estímulo cirúrgico proposto, a droga se mostrou eficaz. A recuperação anes-tésica aconteceu em tempo acima da média, relatada como próxima a 15 minutos [9].

Propofol é um anestésico usado na indução e manutenção de anestesias em cães com segurança, estabilidade e eficiência, mesmo em pacientes debilitados. Devido as suas características, foi a droga de escolha para a anestesia do graxaim, aliando-se à técnica de infusão contínua - método que promove uma estabilidade hemodinâmica, levando a uma diminuição da dose total da droga usada, causando menos efeitos adversos e melhor recuperação $[8,16]$.

As técnicas anestésicas usadas em canídeos silvestres são semelhantes às usadas em cães domésticos, sendo que a anestesia desses pacientes deve incluir segurança e eficácia, ser reversível, oferecer poder de avaliação e de controle da substância, e do custo [3,11]. A partir desses dados, pôde-se concluir que, neste caso, a técnica foi adequada, mostrandose bastante eficaz e segura, podendo-se sugerir seu uso em anestesias no graxaim-do-campo. Uma vez bastante específica, exige uma estrutura adequada e uso de equipamentos especializados, ponto fundamental a ser avaliado antes do procedimento anestésico.

\section{NOTAS INFORMATIVAS}

${ }^{1}$ Zoletil - Virbac do Brasil, Jurubatuba, SP/Brasil.

${ }^{2}$ Dolosal - Cristália Produtos Químicos e Farmacêuticos Ltda., Itapira, SP/Brasil.

${ }^{3}$ Fentanest - Cristália Produtos Químicos e Farmacêuticos Ltda., Itapira, SP/Brasil.

${ }^{4}$ Solução de Ringer com Lactato - Basa Indústria Farmacêutica, Caxias do Sul, RS/Brasil.

${ }^{5}$ Propovan - Cristália Produtos Químicos e Farmacêuticos Ltda., Itapira, SP/Brasil.

${ }^{6}$ Ketofen - Merial Saúde Animal Ltda., Campinas, SP/Brasil.

\section{REFERÊNCIAS}

1 Adetunji A., Ajadi R.A, Adewoye C.O. \& Oyemakinde B.O. 2002. Total intravenous anaesthesia with propofol: repeat bolus versus continuous propofol infusion technique in xylazine - premedicated dogs. Israel Journal of Veterinary Medicine 57: 12p.

2 Aguiar A.J.A., Luna S.P.L., Oliva V.N.L. S. \& Castro G.B. 2001. Continuous infusion of propofol in dogs premedicated with methotrimeprazine. Veterinary Anaesthesia and Analgesia. 28: 220-224. 
3 Aguirre A.A., Principe B., Tannerfeldt M., Angerbjörn A. \& Mörner T. 2000. Field anesthesia of wild artic fox (alopex lagopus) cubs in the swedish lapland using medetimidine-ketamine-atipamezole. Journal of Zoo and Wildlife Medicine. 31: 244-246.

4 Camu F., Lauwers M. \& Vanlersberghe C. 2001. Anestesia Venosa Total. In: White P. F. (Ed). Tratado de Anestesia Venosa. 1.ed. Porto Alegre: Artmed, pp. 370-386.

5 Fantoni D.T. \& Cortopassi S.R.G. 2002. Anestesia de Cães e Gatos. 1. ed. São Paulo: Roca, 389p.

6 Gan T.J. \& Glass P.S. 2001. Anestesia Balanceada. In: White P. F. (Ed). Tratado de Anestesia Venosa. 1. ed. Porto Alegre: Artmed, pp. 343-369.

7 Glowaski M.M. \& Wetmore L.A. 1999. Propofol: application in veterinary sedation and anesthesia. Clinical Techniques in Small Animal Practice. 14: 1-9.

8 Ilkiw J.E. 1999. Balanced anesthesic techniques. Clinical Techniques in Small Animal Practice. 14: 27-37.

9 Keegan R.D. \& Greene S.A. 1993. Cardiovascular effects of a continuous two-hour propofol infusion in dogs. Comparison with isoflurane anesthesia. Veterinary Surgery. 22: 537-43.

10 Lucherini M., Pessino M., Farias A.A. 2004. Canid Specialist Group. Pampas Fox Least Concerm. pp.63-68. Disponível em:<http://www.canids.org>. Acessado em 09/2007.

11 Massone F. 2003. Anestesiologia Veterinária: Farmacologia e Técnicas. 4. ed. Rio de Janeiro: Guanabara Koogan S.A., $252 \mathrm{p}$.

12 Pires J.S., Campello R.A.V., Faria R.X. \& Guedes A.G.P. 2000. Anestesia por infusão contínua de propofol em cães prémedicados com acepromazina e fentanil. Ciência Rural. 30: 829-834.

13 Plumb D.C. 2002. Plumb's Veterinary Drug Handbook. 4. ed. Iowa: Blackwell Publishers, 896p.

14 Ruas J.L., Farias N.A.R., Soares M.P. \& Brum J.G.W. 2003. Babesia sp. em Graxaim do Campo (lycalopex gymnocercus) no Sul do Brasil. Arquivo do Instituto de Biologia de São Paulo. 70: 113-114.

15 Sano T., Nishimura R., Mochizuki, M., Hara Y., Tagawa M. \& SasakI N. 2003. Clinical usefulness of propofol as an anesthesic induction agent in dogs and cats. The Journal of Veterinary Medical Science. 65: 641-643.

16 Short C.E. \& Bufalari A. 1999. Propofol anesthesia. Veterinary Clinics of North America - Small Animal Practice. 29 : 747-778.

17 Stoelting R.K. 1999. Pharmacology \& Physiology in Anesthesic Practice. 3.ed. Philadelphia: Lippincott - Raven, 814p.

18 Tusell J.M., Andaluz A., Prandi D., Costa C. \& Garcia F. 2005. Effects of epidural anaesthesia-analgesia on intravenous anaesthesia with propofol. The Veterinary journal. 169: 108-112. 